\title{
Farmitalia Carlo-Erba-Preisträger 1982-1989
}

Verleihungsjahr

Thema

Preisträger

Verleihungsort

1982

1) Eigenschaften von Hodgkin-Zellinien: Neue pathophysiologische Erkenntnisse zu einer «alten» Erkrankung

2) Untersuchungen zur kurzzeitigen hochdosier-ten Induktionsbehandlung und zur Frage der zyklischen Erhaltungstherapie beim inope-rablen kleinzelligen Bronchialkarzinorn

Diehl/Hannover (Köln)

Kirchner/Hannover

Burrichter/Hannover

Schaadt/Hannover (Köln)

Fonatsch/Hannover

Stein/Kiel (Berlin)

Heit/Ulm

Ziegler/Tübingen

Niederle/Essen

Seeber/Essen (Leverkusen)

Krebskongreß München 1982 Verleihung während der Eröffnung der Deutschen

Krebsgesellschaft am 3.3.1982

1983

la) Hochdosiertes Medroxyprogesteronacetat beim metastasierenden Mammakarzinom -

Beziehung zwischen Krankheitsverlauf und Hormonprofilen -

lb) Studie zur adjuvanten Chemo-(Immuno-) Therapie des Mammakarzinoms

2) Nachweis und Charakterisierung von Calcito-nin-immunreaktiven Proteinen im Serum und Gewebe von Lungentumorpatienten

3) Bone Marrow Biopsies Revisited-A New Dimension for Haematologic Malignancies

Wander/Göttingen Blossey/Göttingen

Schreml/Ulm (Günzburg)

Lang/Ulm

Lohrmann/Ulm (Lemgo)

Betzler/Heidelberg

Schlag/Heidelberg

Luster/Marburg

Gropp/Marburg

Sostman n/Marburg

Havemann/Marburg 
Kalbfleisch/Marburg

Bartl/München

Burkhardt/München

Frisch/Tel Aviv

Anläßlich der Mitgliederversamm-lung der AIO während des Symposiums über

Resistenzprobleme bei der Chemo- und Radiotherapie maligner Tumoren in Regensburg.

Verleihung am 9.6.1983

1984

1) Randomisierte Studie von drei verschiedenen Chemotherapeutikakombinationenbeim metastasierenden Mammakarzinom bei gleichzeitig hormonal behandelten Patientinnen

2) Molekulare Heterogenität von humanem Choriongonadotropin und seinen Unterein-heiten beim Hodenkarzinom

Cavalli/Bellinzona

Pedrazzini/Zürich

Jungi/St. Gallen

Brunner/Bern

Goldhirsch/Bern

Mermillod/Bern

Alberto/Genf

Martz/Zürich

Mann/München

Karl/München

Deutscher Krebskongreß München 1984

Verleihung anläßlich der Mitglieder-versammlung der AIO am 7.3.1984

1985

1) Prognostische Bedeutung von Ploiddie und proliferativer Aktivität bei nicht-kleinzelligen Bronchialkarzinomen

2a) Antineoplastische Wirkung von Alkyl-Lysophospholipid-Derivaten und anderen Äther-

Lipiden - Experimentelle Ergebnisse und

erste klinische Erfahrungen 2b) Die sequentielle Methotrexat-5-Fluor

uracil-Behandlung - ein neuer Ansatz in der

Tumortherapie

Ergebnisse experimenteller und klinischer

Untersuchungen

Volm/Heidelberg

Mattern/Heidelberg

Sonka/Heidelberg

Wayss/Heidelberg

Drings/Heidelberg

Vogt-Moykopf/Heidelberg

Berdel/München

Herrmann/Heidelberg (Berlin)

Osswald/Heidelberg 


\section{Kunz/Heidelberg}

Gemeinsames Symposium der Arbeitsgemeinschaft für Internisti-sche Oonkologie und der Deutschen Gesellschaft für Hämatologie und Onkologie «Knochen-/Knochen-marks-

Metastasen» am 13./14.6.1985inUlm

\section{9}

1986

1) Blut-Hirnschranke und Penetration von Zytostatika

2) Tierexperimentelle Untersuchungen zur the-rapeutischen Wirkung und Toxizität verschiedener lokoregionaler Chemotherapieformen mit 5-Flour-2'desoxyuridin (FUDR) und 5-

Fluorouracil (5-FU) an Lebertumoren

Unger/Göttíngen

Eibl/Göttingen

von Heyden/Einbeck

Krisch/Kiel

Bartkowski/Göttingen

Berger/Heidelberg

Aguiar/Heidelberg

Schlag/Heidelberg

Schmähl/Heidelberg

Herfarth/Heidelberg

Deutscher Krebskongreß

2.-8.3.1986, München

1987

Positive Übereinstimmung zwischen verrin-gerter zellulärer Aufnahme, NADPH-Gluta-thionReduktase-Aktivität und Adriamycin-resistenz bei Ehrlich-Aszites-Tumorzellinien

Ein neuer Beurteilungsparameter zur Bewer-tung von adjuvanten Behandlungen bei postmenopausalen Patientinnen mit operablem Mamma-Ca

Scheulen/Essen

Hoensch/Groß-Gerau

Kappus/Berlin

Seeber/Leverkusen

Schmidt/Essen

Goldhirsch/Bern

Gelber/Boston

SEK-Sympiosium

21./22.3.1987

Heidelberg

1988

1) Pathophysiologie der Hyperprolaktinämie beim Mammakarzinom

2) Zur Behandlung der chronischen myeloi-schen Leukämie mit Interferon Alpha - klini-sche, molekularbiologische und stammzell-physiologische Befunde

Holtkamp/Göttingen

Wuttke/Göttingen 
Nagel/Göttingen

Michel/Göttingen

Rauschecker/Göttingen

Kloke/Essen

Opalka/Essen

Wandl/Essen

Niederle/Essen

Deutscher Krebskongreß 29.2.-4.3.1988 Frankfurt a. M.

1989

Primäre Zytostatika-Resistenz in einem menschlichen Lungentumorxenograft und Oberexpression von DNA-Sequenzen und Membran-Glykoproteinen

Induktion von Schwesterchromatid-Aus-tausch und chromosomalen Aberrationen durch Busulfan bei Philadelphia-Chromosom positiver chronisch myeloischer Leukämie und Normalknochenmark

Volm/Heidelberg

Bak/Heidelberg

Mattern/Heidelberg

Becher/Essen Prescher/Essen

Internationales Symposium der Phase-I/II-Studiengruppe der AIO und der Deutschen Krebsgesellschafte.V. 23724.11. 1989 Frankfurt a. M. 